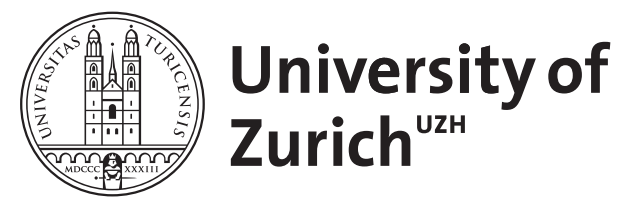

\title{
Shoulder arthroplasty
}

Buck, F M ; Jost, B ; Hodler, J

\begin{abstract}
Shoulder prostheses are now commonly used. Clinical results and patient satisfaction are usually good. The most commonly used types are humeral hemiarthroplasty, unconstrained total shoulder arthroplasty, and semiconstrained inversed shoulder prosthesis. Complications of shoulder arthroplasty depend on the prosthesis type used. The most common complications are prosthetic loosening, glenohumeral instability, periprosthetic fracture, rotator cuff tears, nerve injury, infection, and deltoid muscle dysfunction. Standard radiographs are the basis of both pre- and postoperative imaging. Skeletal scintigraphy has a rather limited role because there is overlap between postoperative changes which may persist for up to 1 year and early loosening and infection. Sonography is most commonly used postoperatively in order to demonstrate complications (hematoma and abscess formation) but may also be useful for the demonstration of rotator cuff tears occurring during follow-up. CT is useful for the demonstration of bone details both pre- and postoperatively. MR imaging is mainly used preoperatively, for instance for demonstration of rotator cuff tears.
\end{abstract}

DOI: https://doi.org/10.1007/s00330-008-1093-8

Posted at the Zurich Open Repository and Archive, University of Zurich ZORA URL: https://doi.org/10.5167/uzh-11349

Journal Article

Published Version

Originally published at:

Buck, F M; Jost, B; Hodler, J (2008). Shoulder arthroplasty. European Radiology, 18(12):2937-2948.

DOI: https://doi.org/10.1007/s00330-008-1093-8 
Florian M. Buck

Bernhard Jost

Juerg Hodler

\section{Shoulder arthroplasty}

because there is overlap between postoperative changes which may persist for up to 1 year and early loosening and infection. Sonography is most commonly used postoperatively in order to demonstrate complications (hematoma and abscess formation) but may also be useful for the demonstration of rotator cuff tears occurring during follow-up. CT is useful for the demonstration of bone details both pre- and postoperatively. MR imaging is mainly used preoperatively, for instance for demonstration of rotator cuff tears.

Keywords Shoulder · Prosthesis · Arthroplasty · Review
B. Jost

Department of Orthopedic Surgery, Orthopedic University Hospital Balgrist,

Zurich, Switzerland

F. M. Buck $(\triangle) \cdot$ J. Hodle
Department of Radiology,

Orthopedic University Hospital Balgrist,

8008 Zurich, Switzerland

e-mail: florian.buck@gmail.com

\begin{abstract}
scintigraphy has a rather limited role now commonly used. Clinical results and patient satisfaction are usually and semiconstained inversed sh der prosthesis. Complications of shoulder arthroplasty depend on the mon complications are prosthetic loosening, glenohumeral instability, periprosthetic fracture, rotator cuff tears, nerve injury, infection, and deltoid muscle dysfunction. Standard radiographs are the basis of both pre-
\end{abstract}

This article reviews the current role of imaging in shoulder arthroplasty. The reader will learn about the different prosthesis types, their indications, contraindications, and complications with special regard to the imaging modalities used.

\section{Indications and contraindications for shoulder arthroplasty}

Osteoarthritis, rheumatoid arthritis, complex fractures of the proximal humerus, osteonecrosis of the humeral head, irreparable tears of the rotator cuff with or without arthropathy ("cuff tear arthropathy"), and revisions of failed prosthesis are the most common reasons to perform shoulder arthroplasty [3]. This method is contemplated when pain and loss of function (mobility, strength) cannot used. Modern prostheses allow the patients to follow an active lifestyle. 
be improved with conservative treatment including analgetics and physiotherapy.

Septic arthritis of the glenohumeral joints and infections in other parts of the body represent absolute contraindications for shoulder replacement surgery. Even successfully treated infections increase the probability of prosthetic infection. Severe bone loss at the glenoid may prevent fixation of the glenoid component.

\section{Prostheses}

\section{Historical development}

Historically, constrained shoulder prostheses were used with the aim of replacing and stabilizing the degenerated glenohumeral joint, based on the assumption that rotator cuff insufficiency or tear had to be compensated by stabilization. This type of prosthesis is no longer in use. Complications included neurovascular injury, loosening, component dissociation, periprosthetic fracture, and ankylosis.

The newer, unconstrained or semiconstrained designs provide far better clinical results. Complications are less common than with constrained implants. They include loosening (mainly of the glenoid component), instability, periprosthetic fracture, rotator cuff tears, and infection.

The outcome after arthroplasty depends on the type of prosthesis, on patient activity, and on the presence of complications. Humeral hemiprostheses implanted before the age of 50 survive more than 10 years in $82 \%$ and more than 20 years in $75 \%$ of patients. Total shoulder arthroplasty appears to be even more successful. After 10 years more than $90 \%$ of patients are satisfied with the result.

\section{Humeral hemiarthroplasty}

Humeral hemiarthroplasty is indicated in avascular necrosis of the humeral head provided the glenoid cartilage is intact (Fig. 1). Hemiarthroplasty can also be used in osteoarthritis when there is a bone-deficient glenoid preventing stable implantation of the glenoid component. Because there is no better alternative humeral hemiarthroplasty is also the treatment of choice in advanced rheumatoid arthritis and other abnormalities with advanced glenoid destruction. In proximal humeral fractures with more than two fragments osteosynthesis may not be successful and there is a risk of avascular necrosis. Therefore, some orthopedic surgeons favor primary hemiarthroplasty over osteosynthesis especially in the elderly with osteoporotic bone. For the success of hemiarthroplasty in humeral fracture stable attachment and healing of the greater and lesser tubercle on the hemiprosthesis is crucial. This requires special prosthetic designs. For a successful outcome humeral length, glenoid retroversion, and the center of rotation of the joint must be restored to the original status.

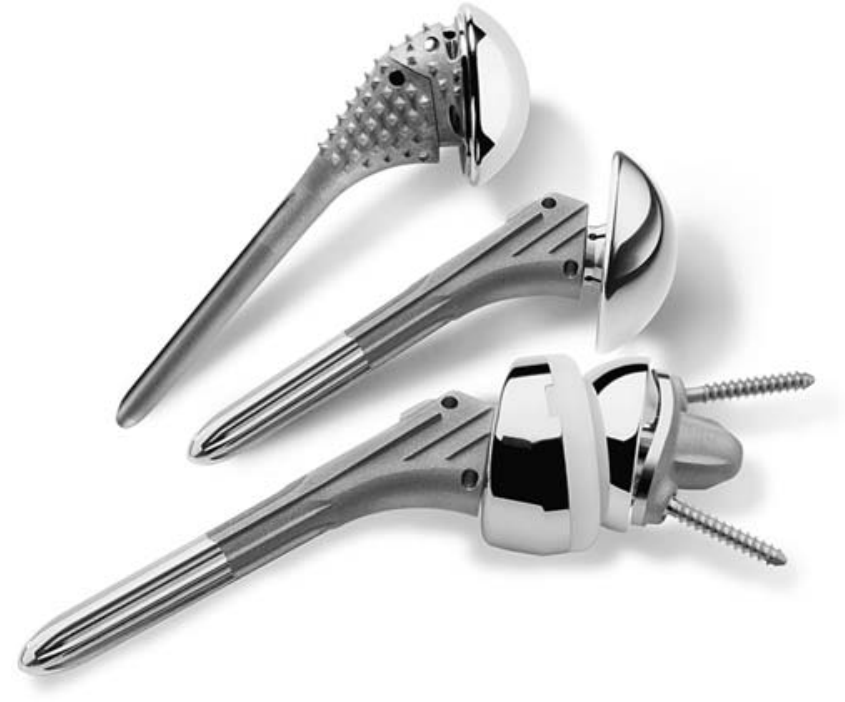

Fig. 1 Examples of the different prosthesis designs most commonly used at the authors' institution (Courtesy Zimmer, Winterthur, Switzerland). Top Unconstrained fracture prosthesis (Anatomical ${ }^{\circledR}$ fracture prosthesis) with a thicker metaphysis with holes for facilitation of refixation of the tuberosities. Middle Unconstrained humeral hemiprosthesis (Anatomical ${ }^{\circledR}$ prosthesis) which can be combined with different glenoid components for total shoulder arthroplasty (Fig. 2). Bottom Semiconstrained inversed total shoulder prosthesis (Anatomical ${ }^{\circledR}$ inversed prosthesis)

\section{Resurfacing}

For resurfacing of the humeral head a shell-like implant replaces the humeral head surface. Anchoring is performed with a short stem. Only minimal resection of the humeral head is performed. There is no need for reaming of the humeral shaft. This type of prosthesis can be combined with a glenoid component. It is mostly employed in young patients with abnormalities limited to the humeral head.

\section{Total shoulder arthroplasty}

Primary and secondary osteoarthritis, as well as early rheumatoid arthritis are the main indications for total shoulder arthroplasty (Figs. 1 and 2). A sufficient rotator cuff and a maintained glenoid (sufficient for successful anchoring of a glenoid component) are required.

\section{Inversed total shoulder prosthesis}

This semiconstrained type of prosthesis is used for irreparable rotator cuff tears with painful loss of shoulder function with or without abnormalities of the glenohumeral joint. Inversed prostheses may also be used for revision surgery. Little is known about the long-term outcome. Currently, they are mainly used in elderly patients. The 
Fig. 2 Two examples of glenoid components for total shoulder prostheses (Courtesy Zimmer, Winterthur, Switzerland). The upper model has four plugs, the lower a keel for fixation. These components are hypodense in conventional radiography and $\mathrm{CT}$ and only visible due to metallic marks (Figs. 3, 4, 7, 8)

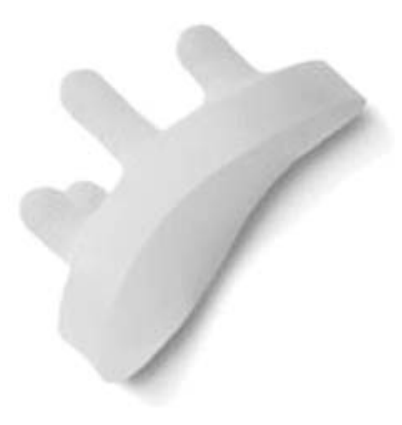

Imaging of shoulder prosthesis

Standard radiography, fluoroscopic examinations

Standard radiographs represent the basis of imaging before and after shoulder arthroplasty. At the authors' institution the standard protocol includes four projections (Fig. 3): (a) An anteroposterior view with the patient rotated approximately $45^{\circ}$ towards the abnormal side (beam parallel to the glenoid surface) and with the tube tilted craniocaudally by $15^{\circ}$. The elbow is flexed $90^{\circ}$ and the hand is pointing towards the x-ray tube (corresponding to slight internal rotation of the glenohumeral joint); (b) an anteroposterior view comparable to (a), but with the forearm in neutral rotation (sagittal plane with reference to the patient's body); (c) a cross-table view; and (d) a Neer's (Y) view with the radiographic beam parallel to the scapula and tilted craniocaudally by $15^{\circ}$.

Standard radiographs do not demonstrate early cartilage damage, but are useful in advanced osteoarthritis of the glenohumeral joints (osteophytes, typically at the inferior humeral head, joint space narrowing, subchondral sclerosis, and cysts in advanced stages). Radiographs also demonstrate cranial migration of the humeral head which is found in advanced rotator cuff tears. Osteoarthritis of the acromioclavicular joint is another radiographic diagnosis. Postoperatively, standard radiographs demonstrate the position of the prosthesis and intraoperative periprosthetic fractures. Later prosthetic loosening, subluxation or dislocation of the prosthesis, and fractures (acute or stressrelated) are of interest.

Fluoroscopy is mainly used for image-guided joint aspirations. Arthrography has a limited role after shoulder
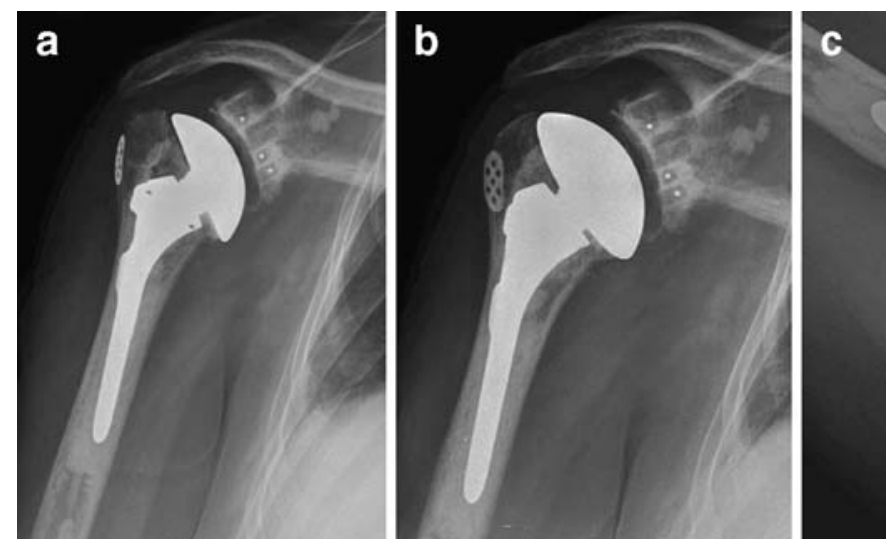

Fig. 3 The four standard projections for standard radiographic evaluation as performed at the author's institution. Example of a patient with anatomical total shoulder arthroplasty. a Anteroposterior view with the patient rotated approximately $45^{\circ}$ towards the abnormal side (beam parallel to the glenoid surface) with the tube tilted craniocaudally by $15^{\circ}$. The elbow is flexed $90^{\circ}$ and the hand is pointing towards the x-ray tube (corresponding to slight internal rotation of the glenohumeral joint). b Anteroposterior view comparable to a, but with the forearm in neutral position (sagittal plane with reference to the patient's body). c Cross-table view. d Neer's (Y) view with the radiographic beam parallel to the scapula and tilted craniocaudally by $15^{\circ}$ 
arthroplasty. It may rarely be used for the demonstration of loosening of the glenoid component, with or without CT (Figs. 4 and 5).

\section{Sonography}

Sonography is mainly used postoperatively for diagnosing hematoma and infection. Sonography is also suitable for image-guided aspiration and drainage and may demonstrate rotator cuff tears and other soft tissue abnormalities during follow-up of the prosthesis.

\section{Skeletal scintigraphy}

Owing to its high negative predictive value, radionuclide imaging is useful for exclusion of prosthetic complications, but has a limited positive predictive value.

Technetium-99m methylene diphosphonate uptake occurs wherever new bone formation takes place [4]. Periprosthetic activity can be identified in most patients for more than 1 year after uncomplicated surgery. The use of gallium-67 scintigraphy and labeled leukocyte scintigraphy improves specificity for infected joint replacement considerably. Publications are rare and do not specifically relate to shoulder arthroplasty $[5,6]$.

\section{Computed tomography}

Computed tomography complements standard radiographs in preoperative planning of shoulder arthroplasty. CT is useful for demonstration of the extent of osteoarthritis, the amount of bone available for fixation, and for measuring glenoid version, for which cross-table standard radiographs

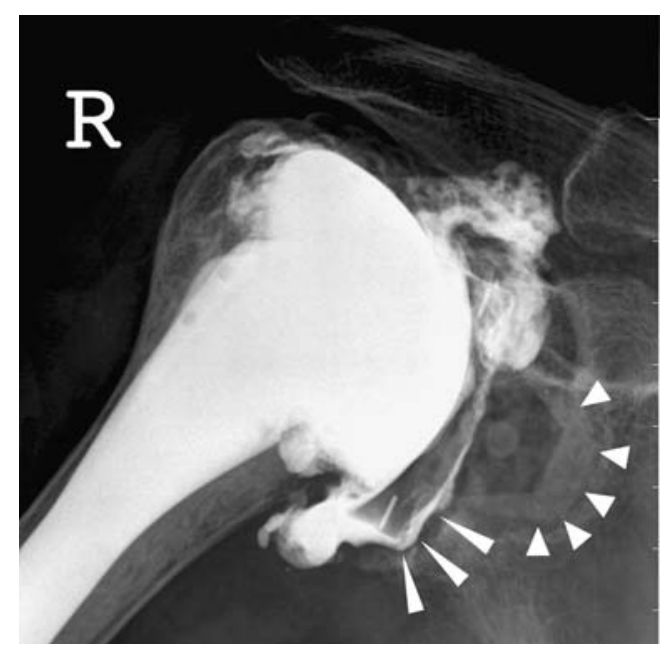

Fig. 4 Arthrography with iodinated contrast between glenoid component and bone (long arrowheads). Normal bone-cement interface at the keel of the glenoid component (short arrowheads)

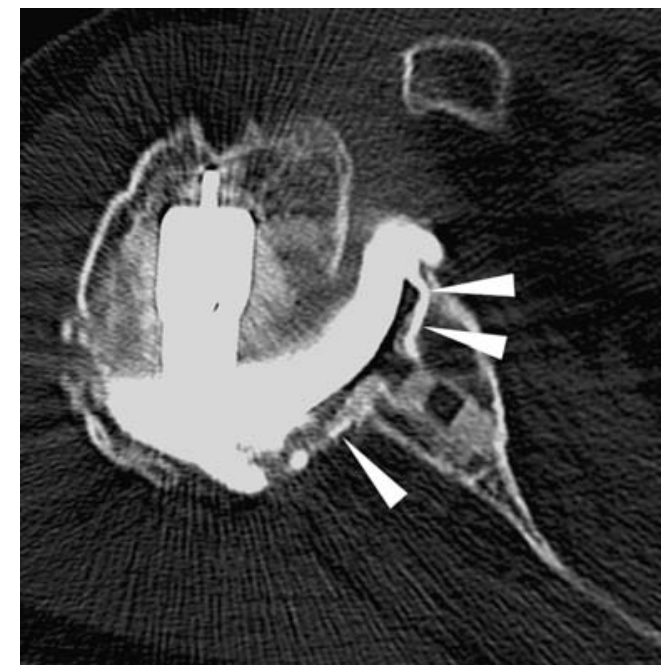

Fig. 5 Computed tomography of the same patient as in Fig. 4 after arthrography. Identical spread of intra-articular injected contrast media with glenoid component loosening (arrowheads)

are not reliable [7]. Glenoid version is typically measured on a thick reconstructed section at the middle of the glenoid. Normally the glenoid articular surface has a minimal retroversion with regard to a line drawn through the middle of the glenoid and the medial scapular margin [8] (mean $3^{\circ}$ retroversion, range $7^{\circ}$ anteversion-16 $6^{\circ}$ retroversion). Humeral head retroversion may be measured using reference lines drawn through the anterior and posterior limits of the humeral head cartilage and through both humeral epicondyles. Humeral head retroversion varies from 9 to $25^{\circ}$, mean $17^{\circ}$ [9]. Fatty degeneration of the rotator cuff muscles is commonly assessed by a grading system published by Goutallier et al. [10] (Table 1). CT is also useful in the detection of periprosthetic fracture of the humerus, stress fractures of the acromion, and the coracoid as well as prosthetic loosening [11] (Fig. 5).

Beam hardening artifacts depend on the diameter, density, and geometry of the metallic implants. Artifacts are proportional to material density. Therefore, cobaltchrome alloy causes more pronounced artifacts than titanium [12]. Artifacts are least pronounced in the direction of shortest diameter of the implant $[13,14]$. Beam hardening artifacts can be reduced with increasing

Table 1 Classification of fatty degeneration of rotator cuff muscles (Goutallier et al.) [10]

Grade of fatty Definition

degeneration

\begin{tabular}{ll}
0 & No fat \\
1 & Streaks of fat \\
2 & Less intramuscular fat than muscle tissue \\
3 & Equal amount of intramuscular fat and muscle tissue \\
4 & More intramuscular fat than muscle tissue \\
\hline
\end{tabular}


tube voltage [15]. Increasing tube current improves the signal-to-noise ratio. Decreasing pitch and slice thickness have a similar effect by increasing effective mAs $[13,16]$. Increased slice thickness of reformatted images, smooth reconstruction filters, and increased window width improve image quality [13]. However, smooth reconstruction filters reduce spatial resolution [15]. Tables 2 and 3 present an imaging protocol optimized for arthroplasty and a checklist for optimization of CT protocols for patients with orthopedic implants.

Intra-articular contrast in the presence of implants is rarely employed. There is some additional information such as joint capsule width and possibly contrast leakage into bone-cement or bone-implant interfaces in loosening.

\section{MR imaging}

MR imaging is mainly used preoperatively for imaging of the rotator cuff tendons (Fig. 6) and muscles (Fig. 7).

Postoperatively, susceptibility artifacts interfere with imaging, but it may still be useful for the assessment of the rotator cuff tendons and muscles, soft tissue hematoma and abscess, or radiographically occult fractures. Different imaging protocols are in use [17-19]. Table 4 provides the standard protocol for postoperative shoulder imaging used at the authors' institution.

Table 2 Suggested CT parameters in arthroplasty

\begin{tabular}{ll}
\hline Parameter & Value \\
\hline FOV & 250 \\
Collimation & 0.625 \\
$\mathrm{kV}$ & 140 \\
Pitch & 0.426 \\
mAs/slice & 300 \\
Matrix & 512 \\
Recon thickness & $0.9 \mathrm{~mm}$ \\
Recon increment & 0.45 \\
Window center & 450 \\
Window width & 2,000 \\
Reconstruction & Axial, coronal, sagittal \\
Additional elbow scan for rotation analysis \\
FOV & 180 \\
Collimation & 1.25 \\
kV & 120 \\
Pitch & N/A, only one rotation \\
mAs & 150 \\
Matrix & 512 \\
Recon thickness & 3.75 \\
Recon increment & 15.00 \\
\hline Paramets a &
\end{tabular}

Parameters are proposed for a 40-row CT scanner adapted to postoperative shoulders with metallic components $F O V$ field of view, $N / A$ not applicable
Table 3 Checklist for improving CT of shoulder prostheses

Increase tube voltage

Increase tube current

Use soft tissue reconstruction algorithm

Assess images with thick slices and use multiplanar reconstruction

Intra-articular contrast is rarely helpful after shoulder arthroplasty. Susceptibility artifacts prevent demonstration of at least part of the contrasted joint. In addition, after arthroplasty, there is an increased risk of infection. Although the probability is still low, the consequences of infection would be severe.

In order to reduce susceptibility artifacts, the implanted prosthesis should be aligned with the main magnetic field $B_{0}[20,21]$ which is typically fulfilled for the stem of the humeral prosthesis. Fast or turbo spin echo sequences reduce susceptibility artifacts in comparison with standard spin echo sequences, particularly if long echo train lengths (ETL) and short interecho spacing are applied [20]. Short tau inversion recovery (STIR) sequences are less susceptible to metallic implants than sequences using frequencyselective fat-suppression [22]. Water excitation sequences are also superior to fat suppression, but may not be available on all scanners [23]. Increasing sampling bandwidth reduces susceptibility artifacts, but increases image blurring. With increasing magnetic field strength $\left(B_{0}\right)$ susceptibility artifacts become more prominent [24]. Artifact reduction may be achieved by shortening TE, increasing matrix in the frequency encoding direction, and decreasing slice thickness [21]. Decreasing voxel size has only a small effect on artifact size, but improves spatial resolution for structures adjacent to the prosthesis [25].

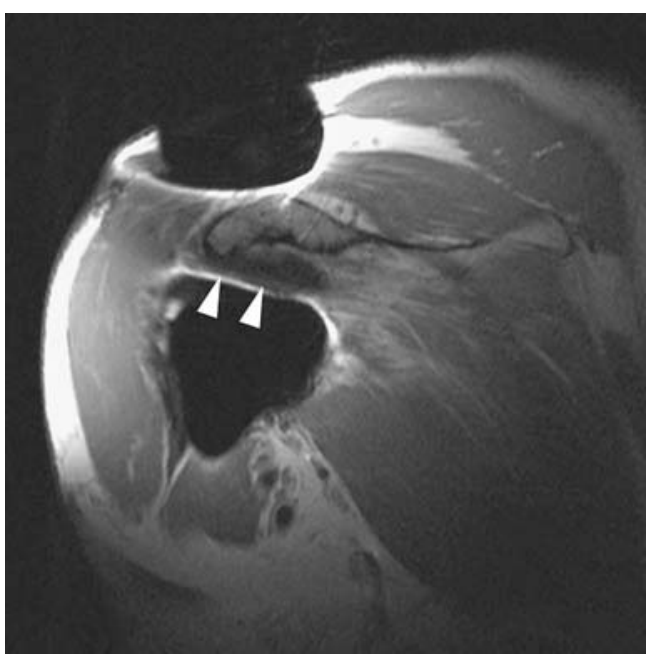

Fig. 6 Coronal proton density (PD)-weighted turbo spin echo image after total shoulder arthroplasty with craniocaudal frequencyencoding direction shows an intact supraspinatus tendon inferior to the acromion (white arrowheads). (TR 2,960 ms, TE $8 \mathrm{~ms}$, ET 9, FOV $18 \times 18 \mathrm{~cm}, 256 \times 212$ matrix, $4-\mathrm{mm}$ slice thickness) 


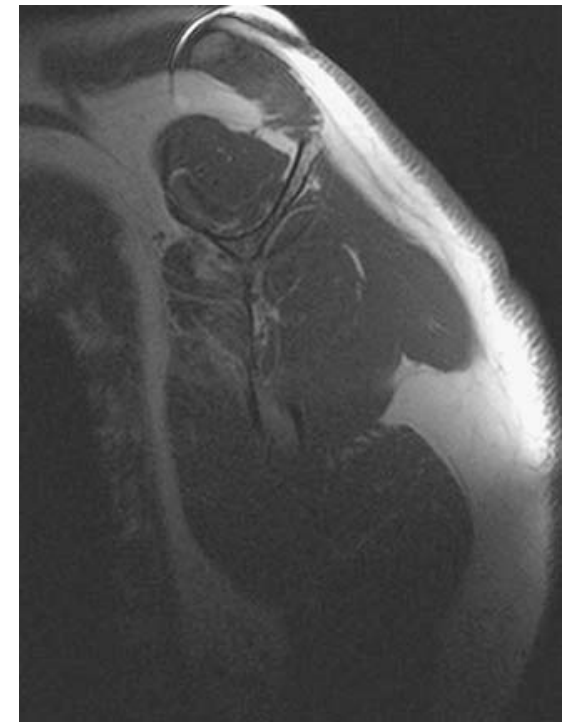

Fig. 7 Sagittal T1-weighted spin echo image after total shoulder arthroplasty with craniocaudal frequency-encoding direction demonstrates rotator cuff musculature and fatty atrophy of subscapularis muscle (Goutallier grade 3) and normal muscle quality of supraspinatus and infraspinatus muscle. (TR $311 \mathrm{~ms}$, TE $5.1 \mathrm{~ms}$, ET 1, FOV $18 \times 18 \mathrm{~cm}, 256 \times 256$ matrix, 5-mm slice thickness)

Suggested MR protocols and a checklist are presented in Tables 4 and 5 .

\section{Complications of shoulder arthroplasty}

Although shoulder arthroplasty is generally successful [26], complications may occur. A revision rate of 7\% after 13.4 years has been reported [27]. Complication rates, patient readmission rates, and duration of hospitalization vary between institutions [28, 29].

A meta-analysis by Bohsali has demonstrated a complication rate of $14.7 \%$ in a series of 2,810 total shoulder replacements [27]. The most common complications were (in order of decreasing frequency): prosthetic loosening, glenohumeral instability, periprosthetic fracture, rotator cuff tears, neural injury, infection, and deltoid muscle dysfunction. Less frequent complications include fracture of the acromion, the scapular spine or the coracoid process, glenoid notching, hematoma, and periarticular soft tissue calcification. Sometimes postoperative persistent shoulder pain or shoulder pain after a longer asymptomatic interval is the leading problem without the presence of the previously mentioned complications. If so, possible causes to be considered are cartilage wear over the glenoid in hemiarthroplasty and synovitis.

\section{Prosthetic loosening}

Prosthetic loosening is the most common complication of shoulder arthroplasty accounting for approximately $40 \%$ of all complications [27, 30]. Loosening typically is diagnosed several years after surgery and predominantly involves the glenoid component. There is an association between glenohumeral instability and glenoid component loosening $[31,32]$. Radiographic signs of loosening are migration (translation and tilting) and the appearance of a radiolucent line at the bone-implant or bone-cement interface [33] (Figs. 8 and 9). Presence of radiolucent lines around the humeral stem can be assessed with a scoring system adapted from the hip [34].

In this system the outline of the humeral prosthesis component is divided into zones numbered from 1 to 7 in anteroposterior and cross-table radiographs. On anteroposterior films zone 1 is located at the proximal third of the prosthesis, laterally, zone 4 at the tip of the prosthesis and zone 7 at the proximal humeral shaft, medially. The same system is applied on cross-table views from anterior to posterior [35]. Sperling et al. defined an additional zone 8 at the base of the head of the humeral component [34].

Glenoid radiolucent lines can be assessed similarly by defining four zones: 1 , around superior baseplate; 2 , around inferior baseplate (without notch if present); 3, around central pillar; 4, around the screws [36].

Lucent lines may be present without clinically significant loosening and do not necessarily correlate with pain [37]. Sanchez-Sotelo and coworkers [38] found that prevalence and extent of humeral radiolucent lines were significantly higher in total shoulder arthroplasty than in hemiarthroplasty. The most reliable radiological evidence

Table 4 Suggested MR parameters in shoulder arthroplasty (1.5 T)

\begin{tabular}{lllll}
\hline Sequence & TR/TE & FOV $(\mathrm{mm})$ & Matrix & Time (min:s) \\
\hline Paracoronal TSE PD-weighted fat sat & $2,640 / 15$ & 160 & $256 \times 512$ & $2: 29$ \\
Paracoronal STIR & $5,590 / 35 / 160$ & 160 & $256 \times 512$ & $3: 34$ \\
Parasagittal STIR & $5,590 / 35 / 160$ & 160 & $256 \times 512$ & $3: 34$ \\
Parasagittal SE T1-weighted & $539 / 15$ & 160 & $256 \times 512$ & $4: 05$ \\
Axial TSE PD-weighted fat sat & $2,640 / 15$ & 160 & $256 \times 512$ & $3: 20$ \\
\hline
\end{tabular}

Section thickness $=4 \mathrm{~mm}$ for all sequences

Abbreviations: FOV field of view, PD proton density, SE spin echo, STIR short tau inversion recovery, Time acquisition time, TR/TE repetition time/echo time, TSE turbo spin echo 
Table 5 Checklist for reduction of implant-related artifacts in MR imaging

Position long axis of prosthesis parallel to the direction of the main magnetic field $\left(B_{0}\right)$

Avoid gradient echo sequences, use spin echo sequences

Use fast/turbo spin echo instead of conventional spin echo sequences

Starting from the prosthesis, the region of interest should not be in the phase-encoding direction

Replace frequency-selective fat-saturated T2-weighted spin echo images by STIR sequence

Reduce echo spacing in fast/turbo spin echo sequences

Increase echo train length

Reduce slice thickness

Increase sampling bandwidth

Increase matrix size (e.g., $512 \times 512$ )

of loosening include radiolucent lines measuring $2 \mathrm{~mm}$ or more around the whole implant as well as subsidence and tilt of the component [34].

Bone resorption at the glenoid can be reduced by preservation of the subchondral bone plate, concentric glenoid reaming [39], and avoiding a mismatch between the radius of the glenoid and the humeral head [40, 41] which increases polyethylene wear.

\section{Notching}

Notching is found in semiconstrained inversed total shoulder arthroplasty. It relates to bone resorption at the

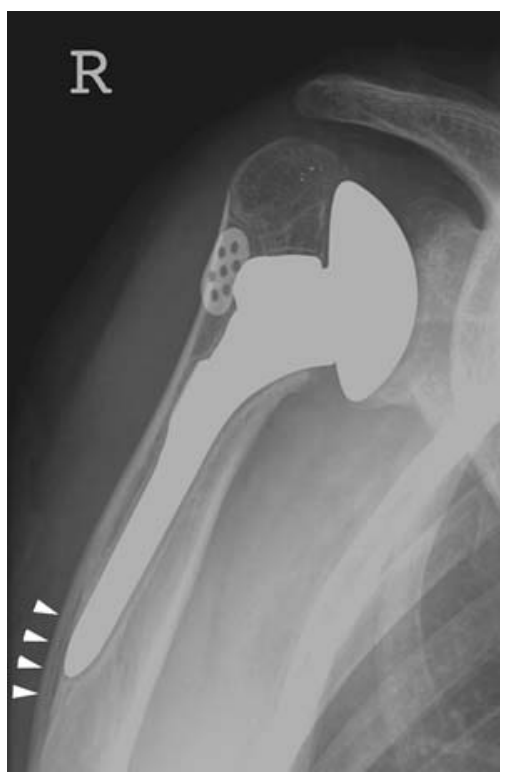

Fig. 8 Loosening of the humeral component: A radiolucent line is visible around the entire humeral implant. Varus tilting of the prosthesis and lateral cortical bulging with periosteal reaction (white arrowheads)

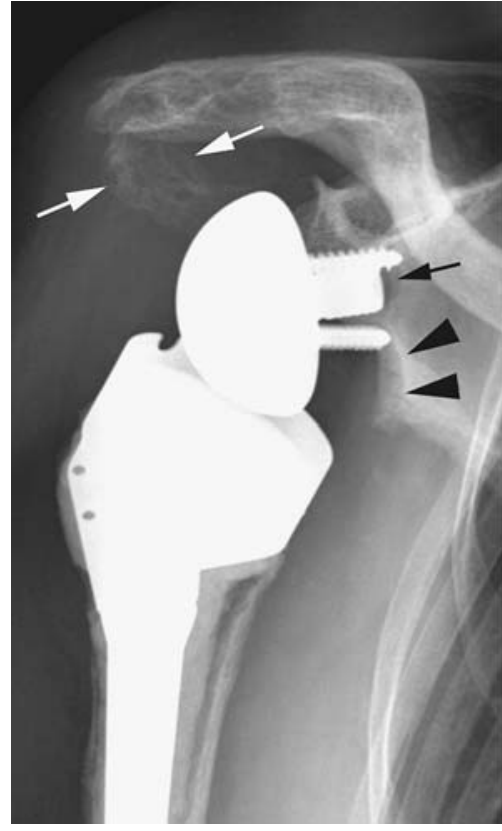

Fig. 9 Fracture and mild inferior dislocation of the acromion (white arrows) after semiconstrained inversed total prosthesis. Notching grade II is noted (black arrowheads) with a radiolucent line (black arrow) at the central pillar of the glenoid component

inferior scapular neck caused by impingement of the humeral cup during adduction (Fig. 9). Notching has been classified as grade 0 (no notch), grade 1 (small notch stopping short of inferior screw), grade 2 (medium notch reaching inferior screw), and grade 3 (large notch extending beyond inferior screw) [36]. Mild notching is not typically symptomatic. However, advanced notching is associated with glenoid component loosening.

\section{Glenohumeral instability}

Glenohumeral instability is the second leading cause of dysfunction after arthroplasty. The prevalence is $4 \%$ which corresponds to $30 \%$ of all complications [27, 30, 31].

Instability after shoulder arthroplasty is typically anterior $(80 \%$ of unstable shoulders). Anterior subluxation of the humeral head by more than $5 \mathrm{~mm}$ as seen on cross-table radiographs or CT suggests anterior instability. Anterior instability may have multiple causes including subscapularis and anterior capsular abnormalities, anteversion of the glenoid component, oversized humeral head component, anterior placement of the humeral component [42], and decreased humeral retroversion $\left(<20^{\circ}\right.$ retroversion $)$. Anterior dislocation may occur when the subscapularis tendon is torn.

The diagnosis of superior instability is made when the acromiohumeral distance is less than $5 \mathrm{~mm}$ on an anteroposterior view. Superior instability is associated with anterior instability, rotator cuff tears [43], cranial 
placement of the humeral component with relative lengthening of the humerus, and superior tilting of the glenoid component.

In posterior instability, a wide dorsal capsule, anterior soft tissue contracture, infraspinatus tendon deficiency, malrotation of humeral component (retroversion $>45^{\circ}$ ), dorsal glenoid defect, and retroversion of glenoid component $\left(>20^{\circ}\right)$ may be responsible.

Inferior instability is typically found when the humerus is shortened which may occur after proximal humeral fractures or humeral defects in tumor surgery. Deltoid muscle weakness also leads to inferior instability. This may be found in axillary nerve palsy or deltoid muscle detachment. Elevation of the arm above the horizontal plane is practically impossible when the humerus is shortened.

Preoperative imaging of the contralateral side for comparison is used for planning of surgery.

\section{Periprosthetic fracture}

Periprosthetic fractures have a prevalence of about $1.5-3 \%$ (11\% of all complications associated with shoulder arthroplasty) [27]. Fractures of the humerus may occur intraoperatively and later due to acute trauma or as a fatigue fracture (Fig. 10). Risk factors include osteoporosis, female sex, increased age, and a tendency to fall. Transverse and short oblique fractures may demonstrate delayed healing or even result in non-union. Different classification systems for periprosthetic fractures are in use (Table 6)

Fig. 10 Example of an antibiotic-impregnated cement spacer after explantation of total shoulder arthroplasty due to infection. Fracture around the spacer is noted
[44]. The most commonly used classification system by Wright and Cofield [3] divides fractures into three types according the position of the fracture in relation to the tip of the humeral component. Groh et al. [45] use similar criteria. Campbell et al. [46] divide fractures according to the position with the humerus. Worland et al. [47] include implant stability.

Treatment of periprosthetic humeral fractures depends on the exact location and course of the fracture. Conservative treatment is preferred in elderly patients with comorbidities. In most cases, surgical treatment with or without replacement of the humeral shaft is necessary [3].

Fractures that are not directly related to the prosthesis

Fractures of the acromion may be found in inversed prosthesis. The mechanism of fracture is unknown. Fatigue fracture due to increased deltoid tension or acute trauma has been discussed. Because surgical fixation of acromial fractures may not be successful and because the fracture is rather well tolerated by the patients conservative treatment of acromion fractures not extending to the scapular spine is preferred at the authors' institution. Acromial fractures can be differentiated from Os acromiale based on their commonly more medial position and the irregular borders, often associated with reactive sclerosis.

Fractures of the coracoid process are even less common than acromial fracture. Repetitive pulling by the conjoined tendon (coracobrachialis, short head of biceps) and impingement of the humeral head may be responsible for acute or chronic coracoid fractures. Treatment is usually conservative.

\section{Rotator cuff tears}

Rotator cuff tears are the fourth most common complication after shoulder arthroplasty [27]. They are relevant for the survival of the implant because asymmetric load of prosthetic components leads to increasing wear and loosening. Postoperative rotator cuff tears are associated with insufficient fixation of the tendon after arthroplasty, oversized prosthesis, malrotation of the humeral component, multiple surgery, aggressive physiotherapy involving external rotation during the early postoperative period, and tendon compromise in humeral lengthening [48-50].

The subscapularis tendon is the most commonly torn tendon after shoulder arthroplasty. Tuberculum minus osteotomy instead of tenotomy can reduce postoperative subscapularis insufficiency considerably [51].

Supraspinatus tears are suspected when the distance between the top of the humeral prosthesis and the acromion is less than $5 \mathrm{~mm}$ on standard radiographs. However, this distance may also appear to be reduced in posterior subluxation of the humeral head after shoulder arthroplasty. 
Table 6 Classification systems for periprosthetic fracture after shoulder arthroplasty

\begin{tabular}{ll}
\hline Classification & Definition \\
\hline Groh et al. [45] & \\
I & Fracture exclusively proximal to the tip of the stem \\
II & Fracture at the tip of the stem, running from proximal of the tip to distal \\
III & Fracture exclusively distal of the tip of the stem \\
Campbell et al. [46] & \\
I & Fracture of the greater or lesser tuberosity \\
II & Fracture of the proximal humeral metaphysis \\
III & Fracture of the proximal humeral diaphysis \\
IV & Fracture of the mid and distal humeral shaft \\
Worland et al. [47] & \\
A & Fracture of the tuberosities \\
B1 & Spiral fracture with stable stem \\
B2 & Short oblique or transverse fracture with stable stem \\
B3 & Any fracture with unstable stem \\
Wright and Cofield [3] & \\
A & Fracture at the tip of the stem extending proximally more than one third the length of the stem \\
B & Fracture at the tip but with less proximal extension \\
C & Fracture distal to the implant and fractures extending into the humeral metaphysis \\
\hline
\end{tabular}

Ultrasonography may be used for the diagnosis of rotator cuff tears. MR imaging may not be able to demonstrate the entire rotator cuff due to the susceptibility artifacts associated with implants. CT may be useful for the demonstration of fatty degeneration of the rotator cuff as a reliable indirect sign of a rotator cuff tear.

\section{Infection}

Postoperative infection is a rare but severe complication of shoulder arthroplasty. Bohsali et al. reported a prevalence of $0.7 \%$ [27]. Periprosthetic infections can occur directly after implantation or with a delayed onset (3 months or later) $[52,53]$. Infection with Staphylococcus aureus is most commonly encountered, followed by coagulasenegative staphylococcus and Propionibacterium acnis. Immunosuppression, diabetes, rheumatoid arthritis and other systemic inflammatory disease, remote infections, previously performed surgery, chemotherapy, corticosteroid medication, and repeated intra-articular steroid injections represent risk factors [31, 54, 55]. Clinical findings, laboratory test, radiography, scintigraphy, and joint aspiration are either insensitive or non-specific, or both [5]. Pain and reduced function are the most common symptoms. Skin reddening and swelling may be present but are frequently not. Laboratory parameters include increased Creactive protein, erythrocyte sedimentation rate, and white blood cell count $[31,52-54]$.

On standard radiographs, infection can only be diagnosed late in the course of disease. Progressive loosening is the most conspicuous sign. Skeletal scintigraphy demon- strates massively increased tracer uptake in infection. However, there is an overlap with normal postoperative uptake which is seen during the first postoperative year. During this same period of time, about two-thirds of prosthetic infections occur. MR imaging and sonography may be useful for the demonstration of soft tissue abnormality associated with implant infection. Immediate revision, aggressive debridement, saving or exchange of the prosthesis depending on the onset of infection, and appropriate intravenous antibiotic therapy is generally rated to be the best treatment, but not feasible in all patients. Two-stage replacement with antibiotic-impregnated cement spacer (Fig. 10) has been employed. Sperling et al. concluded that two-stage reimplantation offers the best outcome [56]. Coste et al. [57] stated that antibiotics or debridement alone are ineffective. Rarely, arthrodesis or even amputation have been used [31, 52-54, 56-62].

Nerve injury/deltoid muscle dysfunction

Intraoperative injury to the brachial plexus and axillary nerve is rare. It leads to deltoid muscle dysfunction with reduced abduction and commonly with inferior instability. Detachment is another reason for deltoid muscle dysfunction. The risk for deltoid muscle detachment depends on the surgical approach. For revision arthroplasty with massive rotator cuff tears deltoid muscle function is crucial. If deltoid muscle function is insufficient not even inversed shoulder prosthesis can be used. Ultrasound and MR imaging may be used for the diagnosis of deltoid muscle abnormalities. 


\section{Heterotopic ossification}

Heterotopic ossification develops early postoperatively [63]. It can be graded according to Kjaersgaard et al. [64], i.e.,by evaluating the space between the medial humeral shaft and the lateral glenoid. Grade 0 means no ossification, grade 1 ossification occupying $<50 \%$ of the space, grade 2 ossification occupying $>50 \%$ of the space, and grade 3 bridging of the space. Ossifications are typically low grade and are often not clinically important [63]. In higher grades joint mobility is reduced. Standard radiographs are typically used for this diagnosis. CT may be used to demonstrate additional details.

\section{Implant failure}

Implant failure is rare. Subluxation or dislocation of polyethylene inlays $[65,66]$, broken fixations screws [67, 68], fracture of the keel or metal glenoid backing [68, 69], and dissociation of the polyethylene glenoid insert from its metal tray $[69,70]$ have been described. Standard radiographs demonstrate part of these complications. CT may provide additional information.

Progressive wear of the glenoid after hemiarthroplasty

After hemiarthroplasty degeneration on the glenoid side of the joint progresses. This is a problem in young, active patients. Surgical revision with total arthroplasty may be necessary. Standard radiographs are typically used for this diagnosis.

\section{Conclusion}

Shoulder prostheses are now widely used. Clinical results and patient satisfaction are good. The most commonly used types are humeral hemiarthroplasty or resurfacing, unconstrained total shoulder arthroplasty, and semiconstrained inversed shoulder prosthesis.

Complications of shoulder arthroplasty depend on the prosthesis type used. The most common complications are prosthetic loosening, glenohumeral instability, periprosthetic fracture, rotator cuff tears, nerve injury, infection, and deltoid muscle dysfunction.

Standard radiographs are the basis of both pre- and postoperative imaging. Skeletal scintigraphy has a rather limited role because there is overlap between postoperative changes which may persist for up to 1 year and early loosening and infection. Sonography is most commonly used postoperatively in order to demonstrate complications (hematoma and abscess formation) but may also be useful for the demonstration of rotator cuff tears occurring during follow-up. CT is useful for the demonstration of bone details both pre- and postoperatively. MR imaging is mainly used preoperatively, for instance for demonstration of rotator cuff tears.

\section{References}

1. Lugli T (1978) Artificial shoulder joint by Pean (1893): the facts of an exceptional intervention and the prosthetic method. Clin Orthop Relat Res 133:215-218

2. Péan JE (1894) Des moyens prosthetiques destinés à obtenir la reparation des parties osseuses. Gaz des Hôp 67:291 Reprinted in English (1973) Clin Orthop 94:4

3. Wright TW, Cofield RH (1995) Humeral fractures after shoulder arthroplasty. J Bone Jt Surg Am 77:1340-1346

4. Palestro CJ, Love C, Miller TT (2006) Infection and musculoskeletal conditions: imaging of musculoskeletal infections. Best Pract Res Clin Rheumatol 20:1197-1218

5. Love C, Tomas MB, Marwin SE, Pugliese PV, Palestro CJ (2001) Role of nuclear medicine in diagnosis of the infected joint replacement. Radiographics 21:1229-1238
6. Pakos EE, Koumoulis HD, Fotopoulos AD, Ioannidis JP (2007) Osteomyelitis: antigranulocyte scintigraphy with 99mTC radiolabeled monoclonal antibodies for diagnosis - meta-analysis. Radiology 245(3):732-741

7. Friedman RJ, Hawthorne KB, Genez BM (1992) The use of computerized tomography in the measurement of glenoid version. J Bone Jt Surg Am 74:1032-1037

8. Nyffeler RW, Jost B, Pfirrmann CW, Gerber C (2003) Measurement of glenoid version: conventional radiographs versus computed tomography scans. J Shoulder Elb Surg 12:493-496

9. Hernigou P, Duparc F, Hernigou A (2002) Determining humeral retroversion with computed tomography. J Bone Jt Surg Am 84-A:1753-1762

10. Goutallier D, Postel JM, Bernageau J, Lavau L, Voisin MC (1994) Fatty muscle degeneration in cuff ruptures. Pre- and postoperative evaluation by CT scan. Clin Orthop Relat Res 304:78-83
11. Ohashi K, El-Khoury GY, Bennett DL, Restrepo JM, Berbaum KS (2005) Orthopedic hardware complications diagnosed with multi-detector row CT. Radiology 237:570-577

12. Haramati N, Staron RB, MazelSperling K et al (1994) CT scans through metal scanning technique versus hardware composition. Comput Med Imaging Graph 18:429-434

13. White LM, Buckwalter KA (2002) Technical considerations: CT and MR Imaging in the postoperative orthopedic patient. Sem Musculoskelet Radiol 6:5-17

14. Vande Berg B, Malghem J, Maldague B, Lecouvet F (2006) Multi-detector CT imaging in the postoperative orthopedic patient with metal hardware. Eur J Radiol 60:470-479

15. Barrett JF, Keat N (2004) Artifacts in CT: recognition and avoidance. Radiographics 24:1679-1691 
16. Wilting JE, Timmer J (1999) Artefacts in spiral-CT images and their relation to pitch and subject morphology. Eur Radiol 9:316-322

17. Zanetti M, Hodler J (2004) MR imaging of the shoulder after surgery. Magn Reson Imaging Clin N Am 12:169-183

18. Owen RS, Iannotti JP, Kneeland JB, Dalinka MK, Deren JA, Oleaga L (1993) Shoulder after surgery: MR imaging with surgical validation. Radiology 186:443-447

19. Gaenslen ES, Satterlee CC, Hinson GW (1996) Magnetic resonance imaging for evaluation of failed repairs of the rotator cuff. J Bone Jt Surg Am 78:1391-1396

20. Suh JS, Jeong EK, Shin KH et al (1998) Minimizing artifacts caused by metallic implants at MR imaging: experimental and clinical studies. AJR Am J Roentgenol 171:1207-1213

21. Port JD, Pomper MG (2000) Quantification and minimization of magnetic susceptibility artifacts on GRE images. J Comput Assist Tomogr 24:958-964

22. Hilfiker P, Zanetti M, Debatin JF, McKinnon G, Hodler J (1995) Fast spin-echo inversion-recovery imaging versus fast T2-weighted spin-echo imaging in bone marrow abnormalities. Invest Radiol 30:110-114

23. Hauger O, Dumont E, Chateil JF, Moinard M, Diard F (2002) Water excitation as an alternative to fat saturation in MR imaging: preliminary results in musculoskeletal imaging. Radiology 224:657-663

24. Merkle EM, Dale BM, Thomas J, Paulson EK (2006) MR liver imaging and cholangiography in the presence of surgical metallic clips at 1.5 and 3 Tesla. Eur Radiol 16:2309-2316

25. Pauchard Y, Smith MR, Mintchev MP (2005) Improving geometric accuracy in the presence of susceptibility difference artifacts produced by metallic implants in magnetic resonance imaging. IEEE Trans Med Imaging 24:1387-1399

26. White CB, Sperling JW, Cofield RH, Rowland CM (2003) Ninety-day mortality after shoulder arthroplasty. J Arthroplasty 18:886-888

27. Bohsali KI, Wirth MA, Rockwood CA Jr (2006) Complications of total shoulder arthroplasty. J Bone Jt Surg Am 88:2279-2292
28. Hasan SS, Leith JM, Smith KL, Matsen FA (2003) The distribution of shoulder replacement among surgeons and hospitals is significantly different than that of hip or knee replacement. J Shoulder Elb Surg 12:164-169

29. Hammond JW, Queale WS, Kim TK, McFarland EG (2003) Surgeon experience and clinical and economic outcomes for shoulder arthroplasty. J Bone Jt Surg Am 85-A:2318-2324

30. Deshmukh AV, Koris M, Zurakowski D, Thornhill TS (2005) Total shoulder arthroplasty: long-term survivorship, functional outcome, and quality of life. J Shoulder Elb Surg 14:471-479

31. Wirth MA, Rockwood CA Jr (1996) Complications of total shoulder-replacement arthroplasty. J Bone Joint Surg Am 78:603-616

32. Gruen TA, McNeice GM, Amstutz HC (1979) "Modes of failure" of cemented stem-type femoral components: a radiographic analysis of loosening. Clin Orthop Relat Res 141:17-27

33. Martin SD, Zurakowski D, Thornhill TS (2005) Uncemented glenoid component in total shoulder arthroplasty. Survivorship and outcomes. J Bone Jt Surg Am 87:1284-1292

34. Sperling JW, Cofield RH, O'Driscoll SW, Torchia ME, Rowland CM (2000) Radiographic assessment of ingrowth total shoulder arthroplasty. J Shoulder Elb Surg 9:507-513

35. Torrens C, Martinez-Diaz S, Ruiz A, Gines A, Caceres E (2007) Assessment of radiolucent lines in cemented shoulder hemi-arthroplasties: study of concordance and reproducibility. Int Orthop doi:10.1007/s00264-007-0452-4

36. Boileau P, Watkinson D, Hatzidakis AM, Hovorka I (2006) Neer Award 2005: The Grammont reverse shoulder prosthesis: results in cuff tear arthritis, fracture sequelae, and revision arthroplasty. J Shoulder Elb Surg 15(5):527540

37. Farron A (2006) Die Schultergelenkprothese. Schweiz Med Forum 6:53-58

38. Sanchez-Sotelo J, O'Discroll SW, Torchia ME, Cofield RH, Rowland CM (2001) Radiographic assessment of cemented humeral components in shoulder arthroplasty. J Shoulder Elb Surg 10:526-531

39. Collins D, Tencer A, Sidles J, Matsen F 3rd (1992) Edge displacement and deformation of glenoid components in response to eccentric loading. The effect of preparation of the glenoid bone. J Bone Jt Surg Am 74:501-507
40. Walch G, Edwards TB, Boulahia A, Boileau P, Mole D, Adeleine P (2002) The influence of glenohumeral prosthetic mismatch on glenoid radiolucent lines: results of a multicenter study. J Bone Jt Surg Am 84-A:2186-2191

41. Iannotti JP, Gabriel JP, Schneck SL, Evans BG, Misra S (1992) The normal glenohumeral relationships. An anatomical study of one hundred and forty shoulders. J Bone Jt Surg Am 74:491500

42. Warren RF, Coleman SH, Dines JS (2002) Instability after arthroplasty: the shoulder. J Arthroplasty 17:28-31

43. Boyd AD Jr, Aliabadi P, Thornhill TS (1991) Postoperative proximal migration in total shoulder arthroplasty. Incidence and significance. J Arthroplasty 6:31-37

44. McDonough EB, Crosby LA (2005) Periprosthetic fractures of the humerus. Am J orthop 34(12):586-591

45. Groh G, Heckman M, Curtis R et al (1994) Treatment of fractures adjacent to humeral prosthesis. In: Annual meeting of the American Academy of Orthopaedic Surgeons. New Orleans, LA

46. Campbell JT, Moore RS, Iannotti JP, Norris TR, Williams GR (1998) Periprosthetic humeral fractures: mechanisms of fracture and treatment options. J Shoulder Elb Surg 7:406-413

47. Worland RL, Kim DY, Arredondo J (1999) Periprosthetic humeral fractures: management and classification. J Shoulder Elb Surg 8:590-594

48. Brems JJ (2002) Complications of shoulder arthroplasty: infections, instability, and loosening. Instr Course Lect 51:29-30

49. Bonutti PM, Hawkins RJ (1992) Fracture of the humeral shaft associated with total replacement arthroplasty of the shoulder. A case report. J Bone Jt Surg Am 74:617-618

50. Miller BS, Joseph TA, Noonan TJ, Horan MP, Hawkins RJ (2005) Rupture of the subscapularis tendon after shoulder arthroplasty: diagnosis, treatment, and outcome. J Shoulder Elb Surg 14:492-496

51. Gerber C, Pennington SD, Yian EH, Pfirrmann CA, Werner CM, Zumstein MA (2006) Lesser tuberosity osteotomy for total shoulder arthroplasty. Surgical technique. J Bone Jt Surg Am 88(Suppl 1 Pt 2):170-177 
52. Wolfe SW, Figgie MP, Inglis AE, Bohn WW, Ranawat CS (1990) Management of infection about total elbow prostheses. J Bone Jt Surg Am 72:198-212

53. Yamaguchi K, Adams RA, Morrey RF (1998) Infection after total elbow arthroplasty. J Bone Jt Surg Am 80:481491

54. Matsen FA 3rd, Rockwood CAJ, Wirth MA, Lippitt SB, Parsons M (2004) Glenohumeral arthritis and its management. In: Rockwood CAJ (ed) The shoulder, 3rd edn. Saunders, Philadelphia, pp 879-1008

55. Rosenberg N, Neuann L, Modi A, Mersich IJ, Wallace AW (2007) Improvements in survival of the uncemented Nottingham total shoulder prosthesis: a prospective comparative study. BMC Musculoskelet Disord 8:76

56. Sperling JW, Kozak TK, Hanssen AD, Cofield RH (2001) Infection after shoulder arthroplasty. Clin Orthop Relat Res 382:206-216

57. Coste JS, Reig S, Trojani C, Berg M, Walch G, Boileau P (2004) The management of infection in arthroplasty of the shoulder. J Bone Jt Surg Br 86:6569
58. Proubasta IR, Itarte JP, Lamas CG, Escriba IU (2005) Permanent articulated antibiotic-impregnated cement spacer in septic shoulder arthroplasty: a case report. J Orthop Trauma 19:666668

59. Ince A, Seemann K, Frommelt L, Katzer A, Loehr JF (2005) One-stage exchange shoulder arthroplasty for periprosthetic infection. J Bone Jt Surg Br 87:814-818

60. Seitz WH Jr, Damacen H (2002) Staged exchange arthroplasty for shoulder sepsis. J Arthroplasty 17:36-40

61. Ramsey ML, Fenlin JM Jr (1996) Use of an antibiotic-impregnated bone cement block in the revision of an infected shoulder arthroplasty. J Shoulder Elb Surg 5:479-482

62. Loebenberg MI, Zuckerman JD (2004) An articulating interval spacer in the treatment of an infected total shoulder arthroplasty. J Shoulder Elb Surg 13:476-478

63. Sperling JW, Cofield RH, Rowland CM (2000) Heterotopic ossification after total shoulder arthroplasty. J Arthroplasty 15:179-182

64. Kjaersgaard-Andersen P, Frich LH, Sojbjerg JO, Sneppen O (1989) Heterotopic bone formation following total shoulder arthroplasty. J Arthroplasty 4:99-104
65. Brenner BC, Ferlic DC, Clayton ML, Dennis DA (1989) Survivorship of unconstrained total shoulder arthroplasty. J Bone Jt Surg Am 71:12891296

66. Clayton ML, Ferlic DC, Jeffers PD (1982) Prosthetic arthroplasties of the shoulder. Clin Orthop Relat Res 164:184-191

67. McElwain JP, English E (1987) The early results of porous-coated total shoulder arthroplasty. Clin Orthop Relat Res 218:217-224

68. Martin SDSC, Thomas WH, Thornhill TS (1995) Total shoulder arthroplasty with an uncemented glenoid component. Paper presented at the annual meeting of the American Shoulder and Elbow Surgeons, Orlando

69. Cofield RHDP (1992) Total shoulder arthroplasty with a tissue-ingrowth glenoid component. J Shoulder Elb Surg 1:77-85

70. Driessnack RP, Ferlic DC, Wiedel JD (1990) Dissociation of the glenoid component in the Macnab/English total shoulder arthroplasty. J Arthroplasty 5:15-18 